\title{
Well-Posedness of the Cauchy Problem for Some Evolution Equations
}

\author{
By \\ Katsuju IGARI*
}

\section{Introduction}

We consider the forward Cauchy problem, in $\mathscr{D}_{L^{2}}^{\infty}$, for the partial differential equation

$$
\begin{aligned}
L[u] & =\partial_{t} u-a\left(x, t ; \partial_{x}\right) u \\
& =\partial_{t} u-\sum_{|\nu| \leqq 2 m} a_{\nu}(x, t) \partial_{x}^{\nu} u=0,
\end{aligned}
$$

$(x, t)$ in $\boldsymbol{R}^{l} \times[0, T], T>0$, with the given initial value at $t=0$, where $m$ is a positive integer. Throughout this paper we use the following abbreviation: $\partial_{t}, \partial_{j}, \partial_{x}^{\nu}$ stand for $\frac{\partial}{\partial t}, \frac{\partial}{\partial x_{j}},\left(\frac{\partial}{\partial x_{1}}\right)^{\nu_{1}} \ldots\left(\frac{\partial}{\partial x_{l}}\right)^{\nu_{l}}, \nu=\left(\nu_{1}, \ldots\right.$, $\left.\nu_{l}\right)$, respectively. We are concerned with the following problem:

Problem. Under what conditions is the above Cauchy problem wellposed?

This problem was studied by many authors. Some of them studied this problem for equations of more general form, namely for $p$-evolution equations. In the case where the coefficients are constant, moreover in the case where the coefficients are functions of only $t$ as well, we know the necessary and sufficient condition for (1.1) to be well-posed. Namely Hadamard's condition, Petrowsky's theorem and so on are well-known. However, in the case where the coefficients are functions of $x$ and $t$, the situation is much more complicated.

One of the most important results already known is the theorem of

Communicated by S. Matsuura, April 25, 1973.

* Department of Mathematics, Kyoto University, Kyoto. 
I. G. Petrowsky and S. Mizohata $([3],[7])$. According to this theorem, we know that the following condition is necessary for (1.1) to be wellposed: for any $x$ in $\boldsymbol{R}^{l}$

$$
\operatorname{Re} a_{2 m}(x, 0 ; i \xi) \leqq 0 \quad \text { for all } \xi \in \boldsymbol{R}^{l},
$$

where $a_{2 m}(x, t ; \xi)$ stands for the principal part of $a(x, t ; \xi)$.

Our purpose of this article is to seek for the more detailed necessary condition. More precisely, we consider the case where for some $x_{0}$ and $\xi^{0}(\neq 0)$ in $\boldsymbol{R}^{l}$

$$
\operatorname{Re} a_{2 m}\left(x_{0}, 0 ; i \xi^{0}\right)=0 .
$$

When $L$ is an operator with constant coefficients, we know by Hadamard's condition that if

$$
\operatorname{Re} a_{2 m-1}\left(i \xi^{0}\right) \neq 0,
$$

the Cauchy problem for (1.1) can not be well-posed in any small neighborhood of $t=0$. Our purpose is to show, assuming that the coefficients of the principal part are real-valued functions, that under certain conditions this is still true (Theorem). We also give the examples which show that without those conditions this is not true (Example 2 and Proposition).

Our main result is Theorem in section 2. Although this theorem is far from complete, however it seems that the condition (C.3) stated there gives a meaningful characterization of the state of degeneracy of the elliptic operator $a\left(x, t ; \partial_{x}\right)$.

On the other hand, we know some sufficient conditions for (1.1) to be well-posed. One of the most useful and well-known equations for which the Cauchy problem is well-posed in $\mathscr{D}_{L^{2}}^{\infty}$ is, of course, the parabolic equation. Recently, the Cauchy problem for degenerate parabolic equations are studied by some authors $([2],[5],[6])$. We should remark that the reasoning shown in $[2]$ is no longer valid in the case $m \geqq 2$.

\section{Theorem and Some Examples}

We say that the forward Cauchy problem for the equation (1.1) in 
$\left.\mathscr{D}_{L^{2}}^{\infty} 1\right)$ is well-posed, if

1) for any given initial value $u_{0}(x) \in \mathscr{D}_{L^{2}}^{\infty}$, there exists a unique solution $u(x, t), t \geqq 0$, which takes the given initial value $u_{0}(x)$ at $t=0$,

2) this linear mapping $u_{0}(x) \rightarrow u(x, t)$ is continuous from $D_{L^{2}}^{\infty}$ into $\mathscr{E}_{t}^{1}\left(\mathscr{D}_{L^{2}}^{\infty}\right)$.

Several examples of equations are known, which satisfy the conditions (C.1) and (C.2), but for which the Cauchy problem is well-posed in $\mathscr{D}_{L^{2}}^{\infty}$.

Example 1 (J. Takeuchi, [9]). Let $L=\partial_{t}-i \partial_{x}^{2}-i a(x) \partial_{x}, x \in \boldsymbol{R}^{1}$.

Suppose that $a(x)$ is a real-valued smooth function with compact support. Then the Cauchy problem for the equation $L u=f$ is well-posed in $\mathscr{D}_{L^{2}}^{\infty}$.

When $a(x)$ is non-zero real constant, it is far from well-posed as Hadamard's condition shows. The reader can find a similar example as the above one in [8]. In view of these examples, it seems difficult to us to find the necessary condition of the pointwise form as (C.2), in general. However, if we assume that the coefficients of the principal part $a_{2 m}(x$, $\left.t ; \partial_{x}\right)$ are real-valued functions, we have the following theorem.

We assume that the coefficients of $a_{2 m}\left(x, t ; \partial_{x}\right)$ are real-valued functions in the class $\mathscr{E}_{t}^{2}\left(\mathscr{B}^{\infty}\right)$, and assume that the other coefficients of $a\left(x, t ; \partial_{x}\right)$ are complex-valued functions in the class $\mathscr{E}_{t}^{0}\left(\mathscr{B}^{\infty}\right)$. By

$$
h_{k}(x ; \xi)=\sum_{|\mu|=k} \frac{1}{\mu !} \partial_{\xi}^{\mu} a_{2 m}(x, 0 ; \xi) \partial_{x}^{\mu} a_{2 m}(x, 0 ; \xi),
$$

we define a homogeneous polynomial $h_{k}(x ; \xi)$ of $\xi$ of degree $4 m-k$.

Theorem. Assume that, for some $x_{0}$ and $\xi^{0}(\neq 0)$ in $\boldsymbol{R}^{l}$,

$$
\begin{aligned}
& a_{2 m}\left(x_{0}, 0 ; i \xi^{0}\right)=0, \\
& \quad \operatorname{Re} a_{2 m-1}\left(x_{0}, 0 ; i \xi^{0}\right) \neq 0,
\end{aligned}
$$

1) In this paper, we use the following linear topological spaces: $\mathscr{D}_{L^{2}}^{\infty}, \mathscr{B}^{\infty}, \mathscr{E}_{t}^{k}\left(\mathscr{D}_{L^{2}}^{\infty}\right)$, $\mathscr{E}_{i}^{k}\left(\mathscr{B}^{\infty}\right)$. We shall explain briefly. $u(x) \in \mathscr{D}_{L^{2}}^{\infty}$ means that all its derivatives $\partial_{x}^{v} u$ (in distribution sense) belong to $L^{2} . \mathscr{D}_{L^{2}}^{\infty}$ provided with the semi-norm $p_{m}(u)=\sum_{|\nu| \leq m}$ $\left\|\partial_{x}^{\nu} u\right\|$ is a Fréchet space. $\varphi(x) \in \mathscr{B}^{\infty}$ means that all its derivatives $\partial_{x}^{\nu} \varphi$ are continuous and bounded. $\mathscr{B}^{\infty}$ provided with the semi-norm $q_{n}(\varphi)=\sum_{|,|} \sup _{x}\left|\partial_{x}^{\prime} \varphi\right|$ is a Fréchet space. $u(x, t) \in \mathscr{E}_{t}^{k}\left(\mathscr{D}_{L^{2}}^{\infty}\right.$ or $\left.\mathscr{B}^{\infty}\right)$ means that $t \rightarrow u(x, t) \in \mathscr{D}_{L^{2}}^{\infty}$ (or $\left.\mathscr{B}^{\infty}\right)$ is continuously differentiable up to order $k$. 


$$
h_{2}\left(x_{0} ; \xi^{0}\right)=0 \text {, }
$$$$
\left(\partial_{t} a_{2 m}\right)\left(x_{0}, 0 ; i \xi^{0}\right) \geqq 0 \text {. }
$$

Then the Cauchy problem for (1.1) is not well-posed in any small neighborhood of $t=0$. Especially in the case $m \geqq 2$, we can remove the condition (C.4).

Note 1. (C.1), (C.2) and (C.4) are invariant under any regular transformation of the space-variables $x$. Moreover, under the fundamental assumption (C.0), the condition (C.3) is invariant, too. The reader will easily verify by considering that $\left(\partial_{x_{j}} a_{2 m}\right)\left(x_{0}, 0, \xi^{0}\right)=0$ for all $j$.

Note 2. We can weaken the assumption for smoothness of the coefficients of the principal part. Namely we need only to assume that the coefficients of $a_{2 m}\left(x, t ; \partial_{x}\right)$ are real-valued functions in the class $\mathscr{E}_{t}^{1+\sigma}\left(\mathscr{B}^{\infty}\right), \sigma>0$.

In our theorem, in general, we can not remove the conditions (C.3) and (C.4). We show this by examples. The following example is related to the condition (C.3).

Example 2. In (1.1), let $a\left(x, t ; \partial_{x}\right)=a\left(x ; \partial_{x}\right)=\partial_{1}^{2}+a\left(x_{1}\right) \partial_{2}^{2}+i b\left(x_{1}\right) \partial_{2}$, $x=\left(x_{1}, x_{2}\right) \in \boldsymbol{R}^{2}$, where $a\left(x_{1}\right)$ and $b\left(x_{1}\right)$ are real-valued functions of only $x_{1}$ in the class $\mathscr{B}^{\infty}$. Assume that $a(0)=0, a\left(x_{1}\right) \geqq 0,\left(\partial_{1}^{2} a\right)(0)>0$. Assume also that for any $\varepsilon>0$ there exists $\delta(>0)$ such that $a\left(x_{1}\right) \geqq \delta$ for any $x_{1} ;\left|x_{1}\right| \geqq \varepsilon$. Then, if

$$
\{b(0)\}^{2}<\frac{1}{2}\left(\partial_{1}^{2} a\right)(0),
$$

the Cauchy problem for the equation $\partial_{t} u-a\left(x ; \partial_{x}\right) u=f$ is well-posed in $\mathscr{D}_{L^{2}}^{\infty}$.

This is the simplest case where $a_{2 m}\left(x_{0}, 0 ; i \xi^{0}\right)=0$ but where $h_{2}\left(x_{0}\right.$; $\left.\xi^{0}\right) \neq 0$. We want to show this by the same reasoning as in [2]. For $u \in \mathscr{D}_{L^{2}}^{2}$,

$$
\begin{aligned}
\left\|\left(\lambda-a\left(x ; \partial_{x}\right)\right) u\right\|^{2}= & \lambda^{2}\|u\|^{2}+\left\|a\left(x ; \partial_{x}\right) u\right\|^{2}+2 \lambda\left(\left\|\partial_{1} u\right\|^{2}+\left\|\sqrt{a\left(x_{1}\right)} \partial_{2} u\right\|^{2}\right) \\
& -2 \lambda \operatorname{Re}\left(u, i b\left(x_{1}\right) \partial_{2} u\right) .
\end{aligned}
$$

Hence, if we show that for $u \in \mathscr{D}_{L^{2}}^{2}$ 


$$
\left\|\partial_{1} u\right\|^{2}+\left\|\sqrt{a\left(x_{1}\right)} \partial_{2} u\right\|^{2}-\operatorname{Re}\left(u, i b\left(x_{1}\right) \partial_{2} u\right) \geqq- \text { const. }\|u\|^{2},
$$

we can get the same proposition as Proposition 2 in [2]. Since

$$
\left(\partial_{1} u, \sqrt{\frac{1}{2} a^{\prime \prime}(0)} x_{1} \partial_{2} u\right)=-\left(u, \sqrt{\frac{1}{2} a^{\prime \prime}(0) \partial_{2}} u\right)+\left(\sqrt{\frac{1}{2} a^{\prime \prime}(0)} x_{1} \partial_{2} u, \partial_{1} u\right),
$$

we have by (2.2),

$$
\left|\left(u, b(0) \partial_{2} u\right)\right|<\left|\left(u, \sqrt{\frac{1}{2} a^{\prime \prime}(0)} \partial_{2} u\right)\right| \leqq\left\|\partial_{1} u\right\|^{2}+\| \sqrt{\frac{1}{2} a^{\prime \prime}(0) x_{1} \partial_{2} u \|^{2} .}
$$

Using a simple partition of unity, we have (2.3) from the above inequality. Other theorems and propositions in [2] can be obtained in the same way as above.

Next we consider the case where $m=1$ and where all of the coefficients are functions of only $t$. Namely we consider the equation

$$
\partial_{t} u-\sum_{j, k=1}^{l} a_{j k}(t) \partial_{j} \partial_{k} u-\sum_{j=1}^{l} b_{j}(t) \partial_{j} u-c(t) u=0
$$

In this case, we can obtain more detailed conditions for well-posedness by Petrowsky's theorem. Assume that the coefficients are continuous, but not assume that $a_{j k}(t)$ are real-valued. The following proposition explains the role of the condition (C.4).

Proposition. Assume that, for some $\xi^{0}(\neq 0)$ in $\boldsymbol{R}^{l}$, there exist positive constants $C$ and $\rho(>2)$ such that

$$
\int_{0}^{t} \operatorname{Re} \sum_{j, k=1}^{l} a_{j k}(s) \xi_{j}^{0} \xi_{k}^{0} d s \leqq C t^{\rho}
$$

in a neighborhood of $t=0$. Then

$$
\operatorname{Im} \sum_{j=1}^{l} b_{j}(0) \xi_{j}^{0}=0
$$

is necessary for (2.4) to be well-posed.

Inversely, if there exists a positive constant $C^{\prime}$ such that

$$
\int_{0}^{t} \min _{|\xi|=1} \operatorname{Re} \sum_{j, k=1}^{l} a_{j k}(s) \xi_{j} \xi_{k} d s \geqq C^{\prime} t^{2}
$$


in a neighborhood of $t=0,(2.4)$ is well-posed in some neighborhood of $t=0$ whatever we may take as lower order terms.

Proof. Apply the Fourier transformation for space-variables $x$ to (2.4). Then

$$
\begin{gathered}
\partial_{t} v(t ; \xi)+\left\{\sum_{j, k=1}^{l} a_{j k}(t) \xi_{j} \xi_{k}-\sum_{j=1}^{l} i b_{j}(t) \xi_{j}-c(t)\right\} v(t ; \xi)=0 . \\
v(t ; \xi)=\exp \left[-\int_{0}^{t}\left\{\sum_{j, k=1}^{l} a_{j k}(s) \xi_{j} \xi_{k}-i \sum_{j=1}^{l} b_{j}(s) \xi_{j}-c(s)\right\} d s\right]
\end{gathered}
$$

is the solution of (2.8), which takes the value 1 at $t=0$. By the theorem of Petrowsky, the Cauchy problem for (2.4) is well-posed for the future if and only if there exist positive constant $C$ and $p$ such that for all $t$, $0 \leqq t \leqq T$, and for all $\xi$ in $\boldsymbol{R}^{l}$

$$
|v(t ; \xi)| \leqq C(1+|\xi|)^{p}
$$

We prove the first half by contradiction. Put $\operatorname{Im} \sum_{j=1}^{l} b_{j}(0) \xi_{j}^{0}=-k$. Without loss of generality, we may assume $k>0$. For $\xi=\tau \xi^{0}$, we have by (2.5)

$$
\left|v\left(t ; \tau \xi^{0}\right)\right| \geqq \exp \left\{\frac{1}{2} k t \tau-C t^{\rho} \tau^{2}\right\}
$$

in a neighborhood of $t=0$. At $t_{\tau}=\left(\frac{1}{4} \frac{k}{C_{\tau}}\right)^{\frac{1}{\rho-1}}$,

$$
\left|v\left(t_{\tau} ; \tau \xi^{0}\right)\right| \geqq \exp \left\{\frac{1}{4} k\left(\frac{k}{4 C}\right)^{\frac{1}{\rho-1} \tau^{\frac{\rho-2}{\rho-1}}}\right\}
$$

Since $\frac{\rho-2}{\rho-1}>0$, this is contradictory to $(2.10)$.

Now we prove the latter half. By (2.7), whatever we may take as lower order terms,

$$
|v(t ; \xi)| \leqq \exp \left\{-C^{\prime} t^{2}|\xi|^{2}+C^{\prime \prime} t|\xi|+C^{\prime \prime \prime} t\right\} \leqq \exp \left\{\frac{1}{4} \frac{C^{\prime \prime 2}}{C^{\prime}}+C^{\prime \prime \prime} T\right\}
$$

Thus (2.10) holds.

Q.E.D. 
Now we give a rough sketch of the proof of Theorem. At first, we remark that we can suppose $x_{0}=0$ and suppose $\xi^{0}=(0, \ldots, 0,1)$, because all of the conditions (C.1) (C.4) are invariant under any regular transformation of space-variables $x$ (Note 1). Moreover we can assume without loss of generality that

$$
\operatorname{Re} a_{2 m-1}\left(0,0 ; i \xi^{0}\right)>0 .
$$

Then consider the operator $L_{0, n}=\partial_{t}-a_{2 m}\left(0,0 ; i n \xi^{0}\right)-\operatorname{Re} a_{2 m-1}\left(0,0 ; i n \xi^{0}\right)$. We shall prove in section 3 that, so far as we restrict the sequence of the initial values in a convenient way, this operator gives a good approximation to $L$. Now we assume that (1.1) is well-posed, then

$$
\max _{0 \leqq t \leqq T}\left\|u_{n}(x, t)\right\|_{L_{x}^{2}} \leqq C \sum_{|\nu| \leqq h}\left\|\partial_{x}^{\nu} u_{n}(x, 0)\right\|_{L_{x}^{2}} .
$$

Finally using the inequality obtained in section 3 , we show that (2.12) is a contradiction.

\section{Operator $L_{0, n}$ Approximating to $L$}

At first, we derive some properties of $a_{2 m}\left(x, t ; \partial_{x}\right)$ from (C. 0$),(\mathrm{C} .1)$ and (C.3). As aforesaid, we can suppose that $x_{0}=0$ and that $\xi^{n}=(0, \ldots$, $0,1)$. Express $a_{2 m}\left(x, t ; \partial_{x}\right)$ as follows:

$$
\begin{aligned}
& a_{2 m}\left(x, t ; \partial_{x}\right)=\sum_{\nu \leqq 2 m-4} a_{\nu}(x, t) \partial_{x}^{\nu}+\sum_{\left|\nu^{\prime}\right|=3} a_{\nu}(x, t) \partial_{x}^{\nu^{\prime}} \partial_{l}^{2 m-3} \\
& +\sum_{j, k=1}^{l-1} a_{j k}(x, t) \partial_{j} \partial_{k} \partial_{l}^{2 m-2}+\sum_{j=1}^{l-1} a_{j}(x, t) \partial_{j} \partial_{l}^{2 m-1}+a(x, t) \partial_{l}^{2 m},
\end{aligned}
$$

where $\nu^{\prime}=\left(\nu_{1}, \ldots, \nu_{l-1}\right)$.

By (C.0) and (C.1), we know that

$$
a(0,0)=0, \quad(-1)^{m} a(x, 0) \leqq 0 .
$$

Therefore by Taylor expansion,

$$
a(x, 0)=\sum_{j, k \leqq 1}^{l} c_{j k} x_{j} x_{k}+r(x), \quad c_{j k}=c_{k j}, r^{(j, k)}(0)=0
$$

for all $j, k=1, \ldots, l$. Now we take an orthogonal matrix $T$ which nor- 
malizes the real symmetric quadratic form $\sum_{j, k=1}^{l-1} c_{j k} x_{j} x_{k}$, and change the space variables by $x=\widetilde{T} x^{\prime}=\left(\begin{array}{ll}T & 0 \\ 0 & 1\end{array}\right) x^{\prime}$. Then

$$
a\left(\widetilde{T} x^{\prime}, 0\right)=\sum_{j=p}^{l-1} c_{j}^{\prime} x_{j}^{\prime 2}+\sum_{j=1}^{l-1} c_{j l}^{\prime} x_{j}^{\prime} x_{l}^{\prime}+c_{l}^{\prime} x_{l}^{\prime 2}+\tilde{r}\left(x^{\prime}\right),
$$

where $p$ is an integer $(1 \leqq p \leqq l),(-1)^{m} c_{j}^{\prime}<0$ for $j=p, \ldots, l-1,(-1)^{m} c_{l}^{\prime} \leqq 0$, $r^{(j, k)}(0)=0$ for all $j, k=1, \ldots, l$. Besides, if we denote $a_{2 m}\left(\widetilde{T} x^{\prime}, t ;{ }^{t} \tilde{T} \partial_{x^{\prime}}\right)$ by $\tilde{a}_{2 m}\left(x^{\prime}, t ; \partial_{x^{\prime}}\right)$, the coefficient of $\partial_{x_{l}^{\prime}}^{2 m}$ of $\tilde{a}_{2 m}\left(x^{\prime}, t ; \partial_{x^{\prime}}\right)$ is $a\left(\widetilde{T} x^{\prime}, t\right)$. Therefore in the expression (3.1) we may assume without the loss of generality that $a(x, 0)$ has the same form as $a\left(\widetilde{T} x^{\prime}, 0\right)$. Namely we may assume that

$$
a(x, 0)=\sum_{j=p}^{l-1} c_{j} x_{j}^{2}+\sum_{j=1}^{l-1} c_{j l} x_{j} x_{l}+c_{l} x_{l}^{2}+r(x)
$$

where $p$ is an integer $(1 \leqq p \leqq l),(-1)^{m} c_{j}<0$ for $j=p, \ldots, l-1,(-1)^{m} c_{l}$ $\leqq 0, r^{(j, k)}(0)=0$ for all $j, k=1, \ldots, l$.

Then by (C.0), (C.1) and (C.3) we can see the following more detailed properties of the coefficients in the expression (3.1). In the following, $\nu^{(1)}$ and $\nu^{(2)}$ stand for $\left(\nu_{1}, \ldots, \nu_{p-1}\right)$ and $\left(\nu_{p}, \ldots, \nu_{l}\right)$ respectively.

$$
a(x, 0)=\sum_{j=p}^{l} c_{j} x_{j}^{2}+\sum_{j=p}^{l-1} c_{j l} x_{j} x_{l}+\sum_{|\nu|=3, \nu(2) \neq 0} c_{\nu} x^{\nu}+r(x)
$$

where $(-1)^{m} c_{j}<0$ for $j=p, \ldots, l-1,(-1)^{m} c_{l} \leqq 0, r^{(\nu)}(0)=0$ for all $\nu$; $|\nu| \leqq 3$.

$$
a_{j}(x, 0)=\sum_{k=p}^{l} c_{k ; j} x_{k}+r_{j}(x), \quad \text { for } j=1, \ldots, p-1
$$

where $r_{j}^{(k)}(0)=0$ for all $k=1, \ldots, l$.

$$
a_{j}(x, 0)=\sum_{|\nu|=2, \nu(2) \neq 0} c_{\nu ; j} x^{\nu}+r_{j}(x), \quad \text { for } j=p, \ldots, l-1,
$$

where $r_{j}^{(\nu)}(0)=0$ for all $\nu ;|\nu| \leqq 2$.

$$
\begin{gathered}
a_{j k}(0,0)=0 \quad \text { for } j \text { or } k=p, \ldots, l-1 \\
a_{j k}(x, 0)=\sum_{k=p}^{l} c_{h ; j, k} x_{h}+r_{j k}(x) \quad \text { for } j \text { and } k=p, \ldots, l-1,
\end{gathered}
$$


where $r_{j k}^{(h)}(0)=0$ for $h=1, \ldots, l$.

$$
a_{\nu}(0,0)=0 \quad \text { for } \nu=\left(\nu^{\prime}, 2 m-3\right) ; \nu^{(1)}=(0, \ldots, 0) .
$$

Proof of (3.3) (3.6). For simplicity, we assume that $m$ is an odd integer. At first, we show (3.3). At the section $x=\left(0, \ldots, x_{j}, 0, \ldots, x_{l}\right)$, $1 \leqq j \leqq p-1, a(x, 0)=c_{j l} x_{j} x_{l}+c_{l} x_{l}^{2}+O\left(\left(x_{j}^{2}+x_{l}^{2}\right)^{3 / 2}\right)$. If we put $x_{j}=\tau x_{l}$ $(-\infty<\tau<\infty)$,

$$
a(x, 0)=c_{j l} \tau x_{l}^{2}+c_{l} x_{l}^{2}+O\left(\left(1+\tau^{2}\right)^{3 / 2} x_{l}^{3}\right) .
$$

If $c_{j l} \neq 0$, putting $\tau=-\left(1+c_{l}\right) / c_{j l}$,

$$
a(x, 0)=-x_{l}^{2}+O\left(x_{l}^{3}\right) \quad \text { in a neighborhood of } x=0 .
$$

This is contradictory to that $a(x, 0) \geqq 0$. Hence $c_{j l}=0$ for $j=1, \ldots, p-1$. By the same way as above, we can see that $c_{\nu}=0$ for $\nu ;|\nu|=3$ and $\nu^{(2)}=0$.

Next we show that $a_{j}(0,0)=0$. At the section $\xi=\left(0, \ldots, \xi_{j}, 0, \ldots, 1\right)$,

$$
a_{2 m}(0,0 ; \xi)=a_{j}(0,0) \xi_{j}+O\left(\xi_{j}^{2}\right)
$$

If $a_{j}(0,0) \neq 0$, it is contradictory to that $a_{2 m}(0,0 ; \xi) \geqq 0$ for all $\xi \in \boldsymbol{R}^{l}$.

Next we show that $a_{j j}(0,0) \geqq 0$ for all $j$. At the section $\xi=(0, \ldots$, $\left.\xi_{j}, 0, \ldots, 1\right)$,

$$
a_{2 m}(0,0 ; \xi)=a_{j j}(0,0) \xi_{j}^{2}+O\left(\xi_{j}^{3}\right)
$$

Hence $a_{j j}(0,0) \geqq 0$, because $a_{2 m}(0,0 ; \xi) \geqq 0$ for all $\xi \in \boldsymbol{R}^{l}$.

Next by the condition (C.3), we show that $a_{j j}(0,0)=0$ for $j=p, \ldots$, $l-1$. In fact

$$
\begin{aligned}
h_{2}\left(0 ; \xi^{0}\right)= & \left.\sum_{|\mu|=2} \frac{1}{\mu !} \partial_{\xi}^{\mu} a_{2 m}(0,0 ; \xi) \partial_{x}^{\mu} a_{2 m}\left(x, 0 ; \xi^{0}\right)\right|_{x=0, \xi=\xi^{0}} \\
= & \frac{1}{2} \sum_{m, n=1}^{l} \frac{\partial^{2}}{\partial_{\xi m} \partial_{\xi n}}\left\{\sum_{\nu \leqq 2 m-3} a_{\nu}(0,0) \xi^{\nu}\right. \\
& \left.+\sum_{j, k=1}^{l-1} a_{j k}(0,0) \xi_{j} \xi_{k} \xi_{l}^{2 m-2}\right\}\left.\frac{\partial^{2}}{\partial x_{m} \partial x_{n}} a(x, 0)\right|_{x=0, \xi=\xi^{0}} \\
= & 2 \sum_{j=p}^{l-1} a_{j j}(0,0) c_{j}=0 .
\end{aligned}
$$


Since $c_{j}>0$ and $a_{j j}(0,0) \geqq 0$ for $j=p, \ldots, l-1$, we see that $a_{j j}(0,0)=0$ for $j=p, \ldots, l-1$. The remaining parts of (3.4) (3.6) can be shown easily by the same arguments as above.

Q.E.D.

Now we want to show that the operator $L_{0, n}=\partial_{t}-a_{2 m}\left(0,0 ; i n \xi^{0}\right)$ $-\operatorname{Re} a_{2 m-1}\left(0,0 ; i n \xi^{0}\right)$ gives, in a certain sense, a good approximation to $L$, where $n$ is a positive large parameter.

Take a function $\beta(x) \in C_{0}^{\infty}, \beta(x) \geqq 0$, which takes the value 1 in $\left\{x ;|x| \leqq \frac{1}{2}\right\}$, and whose support is located in $\{x ;|x| \leqq 1\}$. Define

$$
\beta_{n, \delta}(x)=\beta\left(n^{\frac{1}{4}} \delta^{-1} x_{1}, \ldots, n^{\frac{1}{4}} \delta^{-1} x_{p-1}, n^{\frac{1}{2}} \delta^{-1} x_{p}, \ldots, n^{\frac{1}{2}} \delta^{-1} x_{l}\right),
$$

where $n$ and $\delta$ are large and small positive parameter respectively. Still more take a function $\hat{\alpha}(\xi)$, which takes the value 1 in $\left\{\xi ;\left|\xi-\xi^{0}\right| \leqq \frac{1}{4}\right\}$ and whose support is located in $\left\{\xi ;\left|\xi-\xi^{0}\right| \leqq \frac{1}{2}\right\}$. Define

$$
\hat{\alpha}_{n, \delta}(\xi)=\hat{\alpha}\left(\frac{\xi_{1}}{\delta n^{1 / 2}}, \ldots, \frac{\xi_{p-1}}{\delta n^{1 / 2}}, \frac{\xi_{p}}{\delta n^{3 / 4}}, \ldots, \frac{\xi_{l-1}}{\delta n^{3 / 4}}, \frac{\xi_{l}}{n}\right) .
$$

We denote by $\alpha_{n, \delta}(x)$ the Fourier inverse image of $\hat{\alpha}_{n, \delta}(\xi)$.

At first, apply $\beta_{n, \delta}(x)$ to (1.1),

$$
L\left[\beta_{n, \delta} u\right]=-\sum_{1 \leqq|\nu| \leqq 2 m} \frac{1}{\nu !} \beta_{n, \delta}^{(\nu)}(x) a^{(\nu)}\left(x, t ; \partial_{x}\right) u,
$$

where $a^{(\nu)}\left(x, t ; \partial_{x}\right)$ is defined by $a^{(\nu)}(x, t ; \xi)=\partial_{\xi}^{\mu} a(x, t ; \xi)$. Now it does not change if we modify the coefficients of $a\left(x, t ; \partial_{x}\right)$ outside the support of $\beta_{n, \delta}(x)$, because $L$ acts on $\beta_{n, \delta} u$. Take a function $\zeta(x) \in C_{0}^{\infty}$, which takes the value 1 in $\{x ;|x| \leqq 1\}$, and whose support is located in $\{x$; $|x| \leq 2\}$. Define

$$
\zeta_{n, \delta}(x)=\zeta\left(n^{\frac{1}{4}} \delta^{-1} x_{1}, \ldots, n^{\frac{1}{4}} \delta^{-1} x_{p-1}, n^{\frac{1}{2}} \delta^{-1} x_{p}, \ldots, n^{\frac{1}{2}} \delta^{-1} x_{l}\right) .
$$

We modify $a\left(x, t ; \partial_{x}\right)$ by substituting the coefficients $a_{\nu}(x, t)$ by

$$
\tilde{a}_{\nu}(x, t)=a_{\nu}(0,0)+\zeta_{n, \delta}(x)\left\{a_{\nu}(x, t)-a_{\nu}(0,0)\right\},
$$

and denote it by $\tilde{a}\left(x, t ; \partial_{x}\right)$. Then we can write (3.9) in the form 


$$
\left(\partial_{t}-\tilde{a}\left(x, t ; \partial_{x}\right)\right)\left[\beta_{n, \delta} u\right]=-\sum_{1 \leqq 1} \sum_{\nu ! 2 m} \frac{1}{\nu !} \beta_{n, \delta}^{(\nu)}(x) \tilde{a}^{(\nu)}\left(x, t ; \partial_{x}\right) u .
$$

Next, apply the convolution operator $\alpha_{n, \delta}(x) *$ to (3.12),

$$
\begin{aligned}
& \left(\partial_{t}-\tilde{a}\left(x, t ; \partial_{x}\right)\right)\left[\alpha_{n, \delta} *\left(\beta_{n, \delta} u\right)\right]=\left[\alpha_{n, \delta} *, \tilde{a}\left(x, t ; \partial_{x}\right)\right]\left[\beta_{n, \delta} u\right] \\
& \quad-\alpha_{n, \delta} *\left\{\sum_{1 \leqq 1} \sum_{\nu ! \leqq} \frac{1}{\nu !} \beta_{n, \delta}^{(\nu)}(x) \tilde{a}^{(\nu)}\left(x, t ; \partial_{x}\right) u\right\},
\end{aligned}
$$

where $\left[\alpha_{n, \delta} *, \tilde{a}\left(x, t ; \partial_{x}\right)\right]\left[\beta_{n, \delta} u\right]=\alpha_{n, \delta} *\left\{\tilde{a}\left(x, t ; \partial_{x}\right) \beta_{n, \delta} u\right\}-\tilde{a}\left(x, t ; \partial_{x}\right)$ $\alpha_{n, \delta} *\left\{\beta_{n, \delta} u\right\}$. Denote $\alpha_{n, \delta} *\left(\beta_{n, \delta} u\right)$ and the right-hand term of (3.13) by $v^{(n, \delta)}$ and $f^{(n, \delta)}$ respectively.

Consider now

$$
S_{n, \delta}(t)=\left\|v^{(n, \delta)}(x, t)\right\|_{L_{x}^{2}}^{2}
$$

Hereafter we omit the sufix $(n, \delta)$ of $v^{(n, \delta)}$ and $f^{(n, \delta)}$.

$$
\begin{aligned}
S^{\prime}(t)= & 2 \operatorname{Re}\left(v(t), \partial_{t} v(t)\right)_{L_{x}^{2}} \\
= & 2 \operatorname{Re}\left(v(t), \sum_{|\nu|=2 m} \tilde{a}_{\nu}(x, t) \partial_{x}^{\nu} v(t)\right) \\
& +2 \operatorname{Re}\left(v(t), i \tilde{a}_{2 m-1}^{(2)}(x, t) \partial_{l}^{2 m-1} v(t)\right) \\
& +2 \operatorname{Re}\left(v(t), \tilde{a}_{2 m-1}^{(1)}(x, t) \partial_{l}^{2 m-1} v(t)\right) \\
& +2 \operatorname{Re}\left(v(t), \sum_{\substack{|\nu| \leq 2 m-1 \\
\nu}} \tilde{a}_{\nu}(x, t) \partial_{x}^{\nu} v(t)\right)+2 \operatorname{Re}(v(t), f(t)),
\end{aligned}
$$

where $\tilde{a}_{2 m-1}^{(1)}=\operatorname{Re} \tilde{a}_{2 m-1}, \tilde{a}_{2 m-1}^{(2)}=\operatorname{Im} \tilde{a}_{2 m-1}$.

As aforesaid, we can assume without the loss of generality that

$$
(-1)^{m} \tilde{a}_{2 m-1}^{(2)}(0,0)>0
$$

Since, if we take $n$ and $\delta$ sufficiently large and small respectively, $\left|\tilde{a}_{2 m-1}^{(2)}(x, t)-\tilde{a}_{2 m-1}^{(2)}(0,0)\right|<(-1)^{m} \frac{1}{2} \tilde{a}_{2 m-1}^{(2)}(0,0)$ in a small neighborhood of $t=0$, and since $\operatorname{supp}_{\xi}[v(\xi, t)] \subset \operatorname{supp}_{\xi}\left[\hat{\alpha}_{n, \delta}(\xi)\right]$, we see that

$$
\text { 2-nd term } \geqq 2 c_{0} n^{2 m-1}\|v\|^{2}, c_{0}=\frac{1}{2} \pi^{2 m-1}(-1)^{m} \tilde{a}_{2 m-1}^{(2)}(0,0)>0 .
$$


Besides, by (C.4), (3.3) (3.6) we see that

$$
\left.1 \text {-st term } \geqq-C \delta^{2} n^{2 m-1}\|v\|^{2}, 2\right) \quad \text { for } t ; 0 \leqq t \leqq\left(\delta^{2} n^{-1}\right)^{\frac{3}{4}(2 m-1)} \text {. }
$$

For simplicity, we show this only to $2 \operatorname{Re}\left(v, a(x, t) \partial_{l}^{2 m} v\right)$. In the case of $m=1$,

$$
\tilde{a}(x, t)=\tilde{a}(x, 0)+t \tilde{a}_{t}^{\prime}(0,0)+t\left(\tilde{a}_{t}^{\prime}(x, 0)-\tilde{a}_{t}^{\prime}(0,0)\right)+O\left(t^{2}\right) .
$$

By (3.3) and (3.11) we know that $\sup _{x}|\tilde{a}(x, 0)| \leqq C \delta^{2} n^{-1}$, and that $\sup _{x}\left|\tilde{a}_{t}^{\prime}(x, 0)-\tilde{a}_{t}^{\prime}(0,0)\right| \leqq C \delta n^{-\frac{1}{4}}$. Moreover, by (C.4), $\tilde{a}_{t}^{\prime}(0,0) \leqq 0$. Hence

$$
\begin{aligned}
2 \operatorname{Re}\left(v, \tilde{a}(x, t) \partial_{l}^{2} v\right) & \geqq-\left(C \delta^{2} n+t \tilde{a}_{t}^{\prime}(0,0) n^{2}+C t \delta n^{-\frac{1}{4}} n^{2}+C t^{2} n^{2}\right)\|v\|^{2} \\
& \geqq-C \delta^{2} n\|v\|^{2}, \quad \text { for } t ; 0 \leqq t \leqq\left(\delta^{2} n^{-1}\right)^{3 / 4} .
\end{aligned}
$$

In the case of $m \geqq 2$,

$$
a(x, t)=a(x, 0)+O(t) .
$$

Therefore by (3.3) and (3.11),

$$
2 \operatorname{Re}\left(v, \tilde{a}(x, t) \partial_{l}^{2 m} v\right) \geqq-C\left(\delta^{2} n^{-1}+t\right) n^{2 m}\|v\|_{1}^{2} \geqq-C \delta^{2} n^{2 m-1}\|v\|^{2},
$$

for $t ; 0 \leqq t \leqq\left(\delta^{2} n^{-1}\right)^{\frac{3}{4}(2 m-1)}$. If we note that $\left\|\partial_{x}^{\nu} v\right\| \leqq C \delta^{\left|\nu^{\prime}\right|} n^{\frac{1}{2}|\nu(1)|+\frac{3}{4}|\nu(2)|+\frac{1}{4} \nu l}\|v\|$, we can show (3.18) to the other members in the expression (3.1) by (3.3) (3.6) and (3.11).

Moreover we can see that

$$
\text { 3-rd and } 4 \text {-th terms } \geqq-C n^{2 m-1-\frac{1}{4}}\|v\|^{2} .
$$

Hence, by (3.17) (3.19),

$$
S^{\prime}(t) \geqq\left(2 c_{0} n^{2 m-1}-C \delta^{2} n^{2 m-1}-C n^{2 m-1-\frac{1}{4}}\right)\|v\|^{2}-2\|v\|\|\| f \| .
$$

Thus, taking $\delta$ sufficiently small,

$$
S^{\prime}(t) \geqq c_{0} n^{2 m-1}\|v\|^{2}-C n^{-2 m+1}\|f\|^{2}, \quad 0 \leqq t \leqq\left(\delta^{2} n^{-1}\right)^{\frac{3}{4}(2 m-1)} .
$$

This is the key inequality to prove the theorem. Hereafter we fix the

2) Hereafter we may use the symbol $C$ in order to represent positive constants. Sometimes it expresses a positive constant which can be chosen independently of $n$ and $\delta$. 
parameter $\delta$ and omit it.

\section{Proof of Theorem}

We prove this theorem by contradiction. We assume therefore that (1.1) is well-posed in $\mathscr{D}_{L^{2}}^{\infty}$ in a neighborhood of $t=0$.

At first we define a series of solutions $u_{n}(x, t)$ of (1.1). Namely we define their initial values. Let $\hat{\psi}(\xi)$ be a function whose support is located in $\left\{\xi ;|\xi| \leqq \frac{1}{4}\right\}$. We assume that $\hat{\phi}(\xi) \geqq 0$, and that $\int \hat{\psi}(\xi)^{2} d \xi=1$. We define then

$$
\hat{\psi}_{n}(\xi)=\hat{\phi}\left(\xi-n \xi^{0}\right)
$$

Namely

$$
\psi_{n}(x)=e^{2 \pi i n x_{l}} \psi(x) .
$$

Now we define $u_{n}(x, t)$ by

$$
L\left[u_{n}(x, t)\right]=0, \quad u_{n}(x, 0)=\psi_{n}(x), \quad n=1,2,3, \ldots .
$$

By the assumption, for some $t_{0}(>0), \psi_{n}(x) \rightarrow u_{n}(x, t)$ is continuous from $\mathscr{D}_{L^{2}}^{\infty}$ into $\mathscr{E}_{t}^{1}\left(\mathscr{D}_{L^{2}}^{\infty}\right), 0 \leqq t \leqq t_{0}$. Therefore there exists a positive integer $h$ such that

$$
\max _{0 \leqq t \leqq t_{0}}\left\|u_{n}(x, t)\right\|_{L_{x}^{2} \leqq C n^{h}}
$$

In fact, $\sum_{|\nu| \leqq h}\left\|\partial_{x}^{\nu} \psi_{n}\right\| \leqq C n^{h}$.

Next we want to show that

$$
\left\|\alpha_{n} *\left\{\beta_{n} u_{n}(x, 0)\right\}\right\| \geqq c_{1} n^{-l / 4}, c_{1} \text { is a positive constant. }
$$

Since $\psi(x)$ is analytic, and since $\phi(0)=\int \hat{\psi}(\xi) d \xi>0$, taking $n$ large,

(4.6) $\quad \operatorname{Re} \psi(x) \geqq \frac{1}{2} \psi(0),|\operatorname{Im} \psi(x)| \leqq \frac{1}{4} \psi(0), \quad$ for $x \in \operatorname{supp}\left[\beta_{n}\right]$.

Put $D_{\varepsilon}^{n}=\left\{\xi ;\left|\xi_{j}\right| \leqq \varepsilon n^{\frac{1}{4}}\right.$ for $j=1, \ldots, p-1 ;\left|\xi_{j}\right| \leqq \varepsilon \sqrt{n}$ for $j=p, \ldots, l-1$; $\left.\left|\xi_{l}-n\right| \leqq \varepsilon \sqrt{n}\right\}$. If we take $\varepsilon$ sufficiently small,

$$
\operatorname{Re} e^{-2 \pi i x \cdot\left(\xi-n \xi^{0}\right)} \geqq \frac{1}{2}, \quad \operatorname{Im}\left|e^{-2 \pi i x \cdot\left(\xi-n \xi^{0}\right)}\right| \leqq \frac{1}{4},
$$


for $x \in \operatorname{supp}\left[\beta_{n}\right], \xi \in D_{\varepsilon}^{n}$. Hence for $\xi \in D_{\varepsilon}^{n}$,

$$
\left(\left|\beta_{n} \psi_{n} \hat{)}(\xi)\right| \geqq \operatorname{Re} \int e^{-2 \pi i x \cdot\left(\xi-n \xi^{0}\right)} \beta_{n}(x) \psi(x) d x \geqq \frac{1}{8} \psi(0)\left\{\int \beta(x) d x\right\} n^{-\frac{2 l-p+1}{4}}\right.
$$

Since $\hat{\alpha}_{n}(\xi) \equiv 1$ on $D_{\varepsilon}^{n}$

$$
\begin{aligned}
& \left\|\alpha_{n} *\left(\beta_{n} \psi_{n}\right)\right\|=\| \hat{\alpha}_{n}(\xi)\left(\beta_{n} \psi_{n} \hat{)}(\xi) \| \geqq\left\{\int_{D_{\varepsilon}^{n}} \mid\left(\left.\beta_{n} \psi_{n} \hat{)}(\xi)\right|^{2} d \xi\right\}^{1 / 2}\right.\right. \\
& \quad \geqq \frac{1}{8} \psi(0)\left\{\int \beta d x\right\} n^{-\frac{2 l-p+1}{4}}\left\{\operatorname{vol}\left(D_{\varepsilon}^{n}\right)\right\}^{1 / 2}=c_{1} n^{-\frac{2 l-p+1}{8}} \geqq c_{1} n^{-\frac{l}{4}} .
\end{aligned}
$$

Thus we have (4.5).

Now we consider the right-hand term of (3.13). We want to show that it is expressed as follows:

$$
\begin{aligned}
& f^{(n)}=f^{(n, \delta)}
\end{aligned}
$$

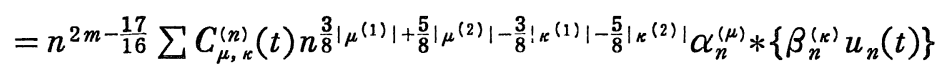

$$
\begin{aligned}
& +O\left(n^{2 m-1-\frac{l+1}{4}}\right)
\end{aligned}
$$

where $\left\|C_{\mu, k}^{(n)}(t)\right\|_{\mathscr{L}\left(L^{2}, L^{2}\right)} \leqq$ constant independent of $n ; 1 \leqq|\mu|+|\kappa| \leqq$ $8 h+2 l+10 ; 0 \leqq t \leqq n^{-\frac{15}{16}(2 m-1)} ; \alpha_{n}^{(\nu)}(x)$ is defined by $\overline{\mathscr{F}}\left[\partial_{\xi}^{\nu} \hat{\alpha}_{n}(\xi)\right] ; \beta_{n}^{(\kappa)}(x)$ $=\partial_{x}^{\kappa} \beta_{n}(x)$.

To see this, take the most delicate part of (3.13), $\left[\alpha_{n} *, \tilde{a}_{\nu}(x, t)\right]$ $\partial_{x}^{\nu} \beta_{n} u_{n},|\nu|=2 m$. Using the Taylor expansion, this can be expressed as

$$
\begin{gathered}
{\left[\alpha_{n} *, \tilde{a}_{\nu}(x, t)\right] \partial_{x}^{\nu} \beta_{n} u_{n}=\sum_{1 \leqq|\mu| \leqq q} \frac{(-1)^{|\mu|}}{\mu !} \tilde{a}_{\nu}^{(\mu)}(x, t)\left(x^{\mu} \alpha_{n}\right) *\left\{\partial_{x}^{\nu} \beta_{n} u_{n}\right\}} \\
+\sum_{|\mu|=q+1} \frac{(-1)^{q+1}}{\mu !} \int \tilde{a}_{\nu, \mu}(x, y, t)(x-y)^{\mu} \alpha_{n}(x-y) \\
\partial_{y}^{\nu}\left[\beta_{n}(y) u_{n}(y, t)\right] d y,
\end{gathered}
$$

where $q=8 h+2 l+10$. Take at first a term of the first part.

$$
\left\|\tilde{a}_{\nu}^{(\mu)}(x, t)\left(x^{\mu} \alpha_{n}\right) * \partial_{x}^{\nu}\left[\beta_{n} u_{n}\right]\right\|
$$

$\leqq$ const. $\sup \left|\tilde{a}_{\nu}^{(\mu)}(x, t)\right| n^{\frac{1}{2}|\nu(1)|+\frac{3}{4}|\nu(2)|+\frac{1}{4} \nu l}\left\|\alpha_{n}^{(\mu)} *\left\{\beta_{n} u_{n}\right\}\right\|$. 
By Leibniz,

$$
\tilde{a}_{\nu}^{(\mu)}(x, t)=\sum_{\mu_{1}+\mu_{2}=\mu} C_{\mu_{1}}^{\mu} a_{\nu}^{\left(\mu_{1}\right)}(x, 0) \zeta_{n}^{\left(\mu_{2}\right)}(x)+t \sum_{\mu_{1}+\mu_{2}=\mu} C_{\mu_{1}}^{\mu} r_{\nu}^{\left(\mu_{1}\right)}(x, t) \zeta_{n}^{\left(\mu_{2}\right)}(x) .
$$

By (3.3),

$$
\begin{aligned}
& \sup \left|\tilde{a}^{(\mu)}(x, t)\right| \leqq C n^{\frac{1}{4}\left|\mu^{(1)}\right|+\frac{1}{2}\left|\mu^{(2)}\right|-1}+C t n^{\frac{1}{4}\left|\mu^{(1)}\right|+\frac{1}{2}\left|\mu^{(2)}\right|} . \quad \text { Hence } \\
& \left\|\tilde{a}^{(\mu)}(x, t)\left(x^{\mu} \alpha_{n}\right) * \partial_{l}^{2 m}\left[\beta_{n} u_{n}\right]\right\|
\end{aligned}
$$

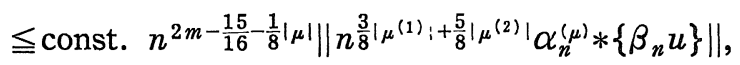

for $0 \leqq t \leqq n^{-\frac{15}{16}(2 m-1)}$. By the same way as above, we can show (4.8) to the other terms by $(3.4) \sim(3.6)$.

Therefore we need only to show that the last part of (4.9) is $O\left(n^{2 m-1-\frac{l+1}{4}}\right)$. These terms are majorized by the form (by Young's theorem)

$$
\begin{aligned}
& \sup \left|\tilde{a}_{\nu ; \mu}^{\left(\nu_{1}\right)}(x, y, t)\right|\left\|\partial_{x}^{\nu}\left(x^{\mu} \alpha_{n}\right)\right\|_{L^{1}}\left\|\beta_{n} u_{n}\right\|_{L^{2}} . \\
& \sup \left|\tilde{a}_{\nu ; \mu}^{\left(\nu_{1}\right)}(x, y, t)\right| \leqq C n^{\frac{1}{4}\left|\mu^{(1)}\right|+\frac{1}{4}|\nu(1)|+\frac{1}{2}\left|\mu^{(2)}\right|+\frac{1}{2} \mid \nu_{1}^{(2) \mid}} .
\end{aligned}
$$

If we note that $\alpha_{n}(x)=n^{\frac{1}{2}(p-1)+\frac{3}{4}(l-p)+1} \alpha\left(n^{\frac{1}{2}} x_{1}, \ldots, n^{\frac{1}{2}} x_{p-1}, n^{\frac{3}{4}} x_{p}, \ldots, n^{\frac{3}{4}} x_{l-1}\right.$, $\left.n x_{l}\right)$,

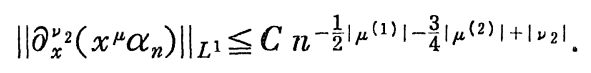

By (4.4), $\left\|\beta_{n} u_{n}\right\| \leqq C n^{h}$. Therefore

$$
(4.10) \leqq C n^{-\frac{1}{4} \mid \mu !+2 m+h} .
$$

Thus we have (4.8).

We consider next all the functions appearing in (4.8). Namely we make the same process for these functions as for $\alpha_{n, \delta} *\left\{\beta_{n, \delta} u_{n}\right\}$ : we replace in the above reasoning $\beta_{n, \delta}(x) \rightarrow n^{-\frac{3}{8}\left|\kappa^{(1)}\right|-\left.\frac{5}{8}\right|_{\kappa} ^{(2)} \mid} \beta_{n, \delta}^{(\kappa)}(x), \alpha_{n, \delta} \rightarrow n^{\frac{3}{8}\left|\mu^{(1)}\right|+\frac{5}{8}\left|\mu^{(2)}\right|}$ $\alpha_{n, \delta}^{(\mu)}$. Then we shall have the equations analogous to (3.13). Consider $S_{n, \delta ; \mu, \kappa}(t)=\left\|\theta_{n, \delta}(\mu, \kappa) u_{n}\right\|^{2}$, where

$$
\theta_{n, \delta}(\mu, \kappa) u_{n}=n^{-\frac{3}{8}\left|\kappa^{(1)}\right|-\frac{5}{8}|\kappa(2)|+\frac{3}{8}\left|\mu^{(1)}\right|+\frac{5}{8}\left|\mu^{(2)}\right|} \alpha_{n, \delta}^{(\mu)} *\left\{\beta_{n, \delta}^{(\kappa)} u_{n}\right\}
$$


Same reasoning as in section 3 will give

$$
S_{n, \delta ; \mu, \kappa}^{\prime}(t) \geqq c_{0} n^{2 m-1} S_{n, \delta ; \mu, \kappa}(t)-C n^{-(2 m-1)}\left\|f^{(n, \delta ; \mu, \kappa)}\right\|^{2},
$$

where $f^{(n, \delta ; \mu, \kappa)}$ are right-hand side of the equations, and can be expressed as

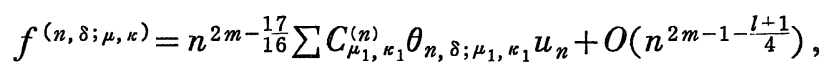

where $\left\|C_{\mu_{1}, \kappa_{1}}^{n}\right\|_{\mathscr{L}\left(L^{2}, L^{2}\right)} \leqq$ const. independent of $n ;|\mu|+|\kappa|+1 \leqq\left|\mu_{1}\right|+$ $\left|\kappa_{1}\right| \leqq 8 h+2 l+10, \mu \leqq \mu_{1}, \kappa \leqq \kappa_{1} ; 0 \leqq t \leqq n^{-\frac{15}{16}(2 m-1)}$.

Finally we define

$$
S_{n}(t)=\sum_{0 \leqq|\mu|+|\kappa| \leqq 8 h+2 l+10}\left\|\theta_{n, \delta ; \mu, \kappa} u_{n}\right\|^{2} .
$$

We have by (3.20), (4.12) and (4.13)

$$
S_{n}^{\prime}(t) \geqq c_{0} n^{2 m-1} S_{n}(t)-C n^{2 m-\frac{9}{8}} S_{n}(t)+O\left(n^{2 m-1-\frac{l+1}{2}}\right) .
$$

Hence we have for large $n$

$$
\begin{aligned}
& S_{n}^{\prime}(t) \geqq \frac{1}{2} c_{0} n^{2 m-1} S_{n}(t)+O\left(n^{2 m-1-\frac{l+1}{2}}\right) . \\
& \left(\exp \left(-\frac{1}{2} c_{0} n^{2 m-1} t\right) S_{n}(t)\right)_{t}^{\prime} \geqq O\left(n^{2 m-1-\frac{l+1}{2}}\right) \exp \left(-\frac{1}{2} c_{0} n^{2 m-1} t\right) .
\end{aligned}
$$

We know, by (4.5) and (4.14),

$$
S_{n}(0) \geqq\left\|\alpha_{n} *\left\{\beta_{n} u_{n}(x, 0)\right\}\right\|^{2} \geqq c_{1}^{2} n^{-\frac{l}{2}} .
$$

By integration of (4.16), we have

$$
S_{n}(t) \geqq \frac{1}{2} c_{1}^{2} n^{-\frac{l}{2}} \exp \left(\frac{1}{2} c_{0} n^{2 m-1} t\right) .
$$

At $t=n^{-\frac{15}{16}(2 m-1)}$

$$
S_{n}\left(n^{-\frac{15}{16}(2 m-1)}\right) \geqq \frac{1}{2} c_{1}^{2} n^{-\frac{l}{2}} \exp \left(\frac{1}{2} c_{0} n^{\frac{1}{16}(2 m-1)}\right) .
$$

On the other hand, since $\left\|\theta_{n ; \mu, \kappa} u_{n}\right\|^{2} \leqq C n^{-\frac{1}{4}|\mu|-\frac{1}{4}|\kappa|+2 h}$, 


$$
S_{n}(t)=O\left(n^{2 h}\right)
$$

Since $c_{0}$ and $c_{1}$ are positive constants, (4.18) and (4.19) can not be compatible. Thus the proof of Theorem is complete.

\section{Acknowledgement}

The author was aroused his interest in this problem through the conversation with Professor Y. Ohya, Professor S. Mizohata encouraged him and gave many useful advices. Especially the method of the proof is due to the one in [3]. The author should like to express his sincere thanks to both professors.

\section{References}

[1] Hasegawa, Y., Strongly p-parabolic systems, Proc. J.A., 37 (1961), 473-477.

[2] Igari, K., Degenerate parabolic differential equations, Publ. RIMS, Kyoto Univ. 9 (1973) 493-504.

[3] Mizohata, S., Some remarks on the Cauchy problem, J. Math. Kyoto Univ. 1-1 (1961), 109-127.

[4] Mizohata, S., Theory of partial differential equations, Iwanami Tokyo (1965) (in Japanese; will appear in English from Camb. Univ. press).

[5] Nagase, M., On the algebra of a class of pseudo-differential operators and the Cauchy problem for parabolic equations, (to appear).

[6] Oleinik, O.A., Linear equations of second order with non-negative form, $M$. Sbornik, 69 (1966) 111-140, (in Russian), Amer. M.S. Transl., 167-199.

[7] Petrowsky, I.G., Über das Cauchysche Problem für ein System linearer partieller Differentialgleichungen im Gebiete der nichtanalytischen Functionen, Bull. de l'Univ. de l'Etat de Moskau, (1938), 1-74.

[8] Strang, G., Necessary and insufficient conditions for well-posed Cauchy problems, J. diff. eq., 2 (1966), 107-114.

[9] Takeuchi, J., Thesis for master's degree, Kyoto Univ. (1969). 
